\title{
Lung ultrasonography as an alternative to chest computed tomography in COVID-19 pneumonia?
}

\author{
Antoine Vieillard-Baron ${ }^{1,2}$, Alberto Goffi, ${ }^{3,4}$ and Paul Mayo ${ }^{5^{*}}$ (1)
}

(C) 2020 Springer-Verlag GmbH Germany, part of Springer Nature

A letter recently published by Yang et al. in this journal raises the important question as to whether lung ultrasonography (LUS) may be an useful alternative to chest computed tomography (CT) for the evaluation of COVID-19 pneumonia [1]. The information in the study is particularly relevant to a situation in which an overwhelming volume of COVID-19 patients may exceed $\mathrm{CT}$ performance and processing capacity. What is new and provocative in Yang's study is the reported higher sensitivity of LUS compared to CT for the detection of alveolar-interstitial syndrome (AIS), consolidation, and pleural effusion in patients with COVID-19 pneumonia. The authors found weak or very weak agreement between LUS and CT for alveolar-interstitial findings, consolidation, and pleural effusion, with CT not able to identify a significant proportion of these findings. This editorial comments on the controversy engendered by the letter with the intent of furthering the discussion on LUS and $\mathrm{CT}$ as imaging modalities for SARS-CoV-2 pneumonia.

Lung ultrasonography offers the clinician an alternative imaging modality to CT for management of COVID-19. We already know that LUS is more accurate than chest radiography to detect pneumothorax, pleural effusion, AIS, and consolidation [2, 3]. In acute respiratory distress syndrome (ARDS), LUS has been reported to be effective for evaluating the extent of pulmonary edema [4] and identifying poorly aerated areas [5]. LUS also allows for assessment of the effects of prone position [6] and positive end-expiratory pressure on lung re-aeration [7],

\footnotetext{
*Correspondence: mayosono@gmail.com

${ }^{5}$ Division of Pulmonary, Critical Care, and Sleep Medicine, Northwell LIJ/ NSUH, New Hyde Park, NY, USA
}

Full author information is available at the end of the article although it does not identify over-inflation. For all these reasons, it is reasonable to consider LUS as useful for the management of COVID-19 patients with ARDS (Table 1).

How is it possible that LUS might be a more sensitive test than CT for detection of findings that are typical of COVID-19 pneumonia? Two key aspects of the study conducted by Yang et al. may explain the high sensitivity described by the authors: misalignment between the definitions used to describe LUS and CT findings and the lack of a reference standard. An alveolar-interstitial pattern was reported in $60 \%$ of LUS images versus in only $38.5 \%$ of CT areas. Yang et al. equated the presence of more than three B-lines to the exclusive presence of ground glass opacities (GGOs) on CT. This decision may explain the lack of correlation between the two imaging modalities. The GGOs seen on CT in COVID-19 are associated with coalescent B line pattern (light beams"), whereas discrete $B$ lines may be associated with other findings on $\mathrm{CT}$ such as interstitial abnormalities [8,9]. A similar definition misalignment may have occurred in relation to lung consolidations, which were reported in only $3 \%$ of CT versus $38.9 \%$ of LUS regions. No definition was provided for lung consolidations in the original manuscript, which was published within the constraints of a short letter. LUS also identified more pleural effusions $(n=67)$ than did CT $(n=14)$. Is the higher sensitivity of LUS for pleural effusions clinically relevant? It is reasonable to assume that most of the pleural effusions detected exclusively with LUS were very small and localized, as suggested in Fig. 1 of the Yang article, so clinically relevant pleural effusions were, therefore, not likely to have been missed by CT. In addition, CT does not always differentiate between pleural effusion, pleural thickening, or lung atelectasis/consolidation. 
Table 1 Comparison of chest CT and lung ultrasonography for evaluation COVID-19 pneumonia

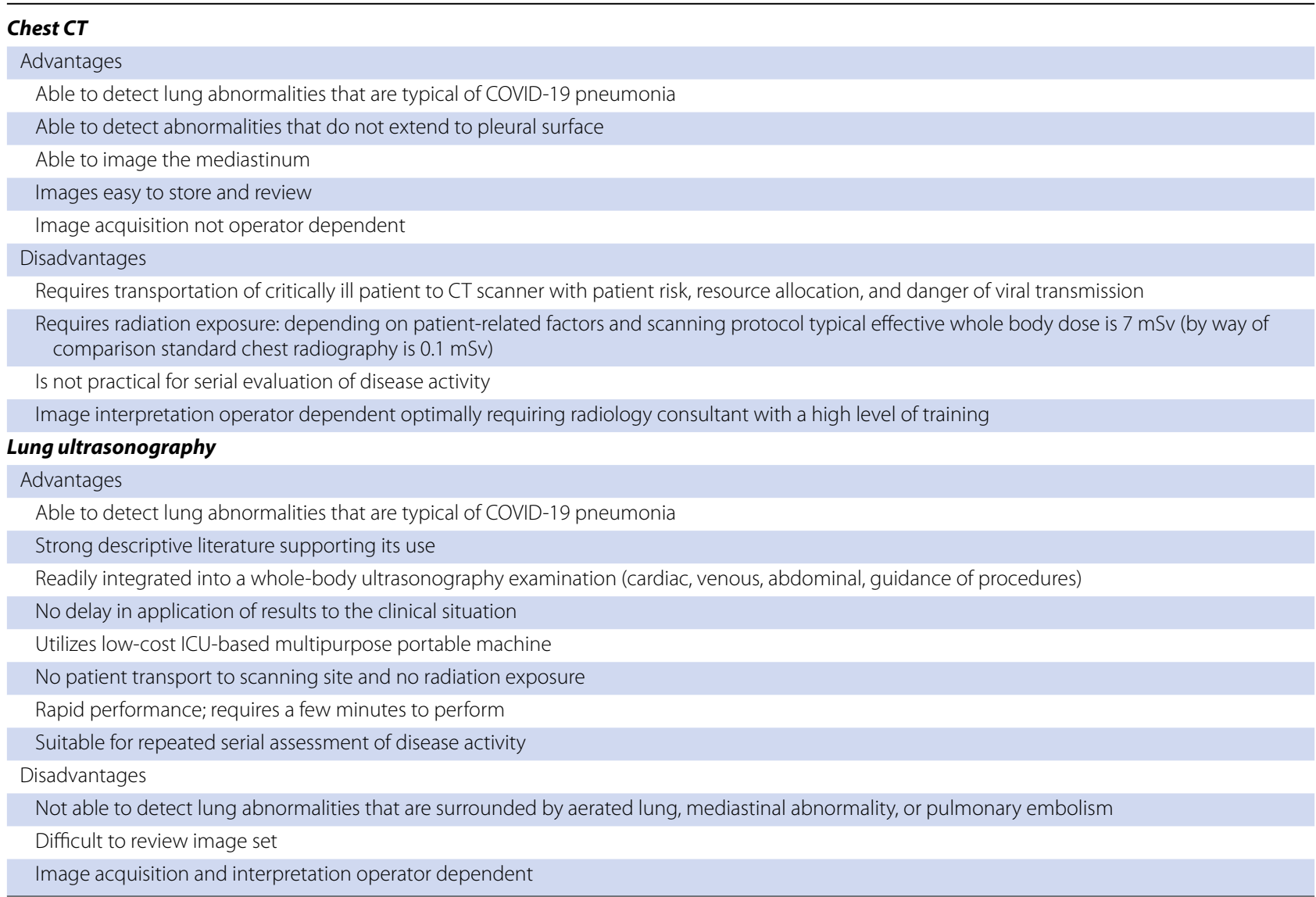

While Yang et al. claimed that their findings suggest LUS is more sensitive than CT for detection of pathological lung processes in patients with COVID-19, we believe that the only conclusion that can reasonably be drawn from their cohort of COVID-19 patients is that LUS assessment identified findings that suggest disease involvement in more lung regions than did CT. However, lacking a gold standard (e.g. pathology), it is impossible to ascertain whether these results reflect a higher rate of false positive findings on LUS (i.e. less specificity) or a higher rate of false negative findings on CT (i.e. greater sensitivity).

We are not sure these findings could be replicated in critically ill patients. As stated by the authors, patients with severe COVID-19 pneumonia, with poor acoustic windows, and with known pre-existent lung pathology were excluded from this study. It is likely that critically ill patients with severe COVID lung involvement will have extensive findings on both LUS and CT. This would result in good agreement between the two techniques.

Finally, this preliminary report, provocative and hypothesis-generating, needs to be confirmed (or not) in a larger population of critically ill patients, with standardized semiology. We also want to emphasize that LUS requires adequate training; and, when performed with attention to details, it can be time consuming. This could potentially prolong the period of time clinicians are exposed to SARS-CoV-2 at the bedside. These disadvantages are balanced against the ease of use of LUS, its serial repeatability, its low cost, its lack of radiation exposure, and its logical integration with other aspects of critical care ultrasonography. It remains to determine how to best combine LUS and CT for management of COVID-19.

\footnotetext{
Author details

${ }^{1}$ University Hospital Ambroise Pare, Assistance-Publique des Hôpitaux de Paris, Paris, France. ${ }^{2}$ INSERM, UMR 1018, Team 5 Kidney-Heart, Centre de Recherche en Epidémiologie Et Santé Des Populations, Villejuif, France. ${ }^{3}$ Interdepartmental Division of Critical Care Medicine and Department of Medicine, University of Toronto, Toronto, ON, Canada. ${ }^{4}$ Department of Medicine, Division of Critical Care Medicine, St. Michael's Hospital, Toronto, ON, Canada. ${ }^{5}$ Division of Pulmonary, Critical Care, and Sleep Medicine, Northwell LIJ/NSUH, New Hyde Park, NY, USA.
}

\section{Compliance with ethical standards}




\section{Conflicts of interest}

The authors declare that they do not have any conflict of interest.

\section{Publisher's Note}

Springer Nature remains neutral with regard to jurisdictional claims in published maps and institutional affiliations.

Received: 8 July 2020 Accepted: 17 August 2020

Published online: 25 August 2020

\section{References}

1. Yang Y, Huang Y, Yuan L, Wang Z (2020) Lung ultrasonography versus chest CT in COVID-19 pneumonia: a two-centered retrospective comparison study from China. Intensive Care Med 25:1-3

2. Mayo PH, Copetti R, Feller-Kopman D, Mathis G, Maury E, Mongodi S, Mojoli F, Volpicelli G, Zanobetti M (2019) Thoracic ultrasonography: a narrative review. Intensive Care Med 45:1200-1211

3. Tierney DM, Huelster JS, Overgaard JD, Plunkett MB, Boland LL, St Hill CA, Agboto VK, Smith CS, Mikel BF, Weise BE, Madigan KE, Doshi AP, Melamed RR (2020) Comparative performance of pulmonary ultrasound, chest radiograph, and $\mathrm{CT}$ among patients with respiratory failure. Crit Care Med 48:151-157
4. Baldi G, Gargani L, Abramo A, D'Errico L, Caramella D, Picano E, Giunta F, Forfori F (2013) Lung water assessment by lung ultrasonography in intensive care: a pilot study. Intensive Care Med 39:74-84

5. Volpicelli G, Elbarbary M, Blaivas M, Lichtenstein D, Mathis G, Kirkpatrick A, Melniker L, Gargani L, Noble V, Via G, Dean A, Tsung JW, Soldati G, Copetti R, Bouhemad B, Reissig A, Agricola E, Rouby JJ, Arbelot C, Liteplo A, Sargsyan A, Silva F, Hoppmann R, Breitkreutz R, Seibel A, Neri L, Storti E, Petrovic T (2012) International liaison committee on lung ultrasound (ILCLUS) for international consensus sonference on lung ultrasounf (ICC-LUS). Int Evid Based Recomm Point Care Lung Ultrasound 38:577-591

6. Haddam M, Zieleskiewicz L, Perbet S, Baldovini A, Guervilly C, Arbelot C, Noel A, Hammad E, Antonini F, Lehingue S, Peytel E, Lu Q, Bouhemad B, Golmard JL, Langeron O, Martin C, Muller L, Rouby JJ, Constantin JM, Papazian L, Leone M, CAR'Echo collaborative network AzuRea collaborative network (2016) Lung ultrasonography for assessment of oxygenation response to prone position ventilation in ARDS. Intensive Care Med 42:1546-1556

7. Bouhemad B, Brisson H, Le-Guen M, Arbelot C, Lu Q, Rouby JJ (2011) Bedside ultrasound assessment of positive end-expiratory pressure-induced lung recruitment. Am J Respir Crit Care Med 183:341-347

8. Volpicelli G, Gargani L (2020) Sonographic signs and patterns of COVID19 pneumonia. Ultrasound J 12:22

9. Volpicelli G, Lamorte A, Villén T (2020) What's new in lung ultrasound during the COVID-19 pandemic. Intensive Care Med 46:1445-1448 